\title{
Numerical modelling of multi-vane expander operating conditions in ORC system
}

\author{
Józef Rak ${ }^{1}$, Przemysław Błasiak ${ }^{1}$, and Piotr Kolasiński ${ }^{1, *}$ \\ ${ }^{1}$ Wrocław University of Science and Technology, Department of Thermodynamics, Theory of \\ Machines and Thermal Systems, Wybrzeże Wyspiańskiego 27, 50-370 Wrocław, Poland
}

\begin{abstract}
Multi-vane expanders are positive displacement volumetric machines which are nowadays considered for application in micro-power domestic ORC systems as promising alternative to micro turbines and other volumetric expanders. The multi-vane expander features very simple design, low gas flow capacity, low expansion ratios, an advantageous ratio of the power output to the external dimensions and are insensitive to the negative influence of the gas-liquid mixture expansion. Moreover, the multi-vane expander can be easily hermetically sealed, which is one of the key issues in the ORC system design. A literature review indicates that issues concerning the application of multi-vane expanders in such systems, especially related to operating of multi-vane expander with different lowboiling working fluids, are innovative, not fully scientifically described and have the potential for practical implementation. In this paper the results of numerical investigations on multi-vane expander operating conditions are presented. The analyses were performed on threedimensional numerical model of the expander in ANSYS CFX software. The numerical model of the expander was validated using the data obtained from the experiment carried out on a lab test-stand. Then a series of computational analysis were performed using expanders' numerical model in order to determine its operating conditions under various flow conditions of different working fluids.
\end{abstract}

\section{Introduction}

Organic Rankine Cycle systems (ORCs) are the Clausius-Rankine (CR) vapour power plants adopting low boiling working fluids. Its operation principle is the same as classic CR systems, however the system design is different. ORCs utilize smaller heat exchangers instead of steam boiler and condenser. Also, a gas turbine or volumetric expander is applied instead of steam turbine. Compared to CR systems, ORCs feature smaller overall dimensions and system power. ORC systems provide the opportunity for heat harvesting from renewable and waste sources featuring low $\left(40-250{ }^{\circ} \mathrm{C}\right)$ and medium temperature $\left(250-500{ }^{\circ} \mathrm{C}\right.$ ) of the carrier (such as e.g. solar, geothermal or industrial waste heat) and convert this energy into electricity, useful heat and cool. Research and development works

\footnotetext{
*Corresponding author: piotr.kolasinski@pwr.edu.pl
} 
on ORC systems are currently proceeding in a number of R\&D and scientific institutions around the world. As the diversification of the energy supply is nowadays of great interest especially the design of small- and micro-power ORC systems is one of the most actively developed research topics. Despite numerous studies, reliable and safe small- and micropower ORC systems, possible for application in e.g. domestic applications, are still not fully developed.

A literature review $[1,2]$ shows that the ORC systems can be classified according to different criteria. By temperature of the heat source, they can be classified into systems powered by high-, medium-, and low temperature sources. By power, ORCs can be classified into systems featuring high power (500 kW or more), medium power (100-500 $\mathrm{kW})$, small power (10-100 kW) and micro power (0.5-10 kW). By the type of the applied expander, ORCs can be classified into systems adopting turbines or systems adopting volumetric expanders.

Small- and micro-power ORC systems dedicated to domestic use by the prosumers utilize heat sources featuring low thermal power and low carrier temperature. Temperature and output characteristics of these sources are often floating, which translates into adverse and changing conditions of the heat supply, and negatively influences the ORC system operation. In small- and micro-power ORCs mainly volumetric expanders are applied (e.g. scroll expanders). However, there are also attempts to adopt micro turbines into these systems. Domestic ORC systems are still under research and development, there are no commercially viable solutions currently available and the majority of these systems are at the level of lab-prototypes. By the technical configuration, these systems can be classified into power plants and CHPs (Combined Heat and Power). Ongoing research and development works on small- and micro-power ORCs are focused on the selection of working fluid, heat exchangers, pumps and expanders design. Small- and micro-power ORC systems designed for domestic use by prosumers should be cheap and reliable. The main part of the ORC system cost is the price of heat exchangers and the expander. The micro turbine design requires high precision workmanship that directly translates into the high price of such machines. In order to provide optimum operating conditions and high efficiency of the micro turbine, the working medium flow through the machine should be maintained on a high and stable level. Such flow conditions can be obtained only if special construction high-output pumps are applied. In small and micro-power domestic ORC systems it is necessary to minimize the power and external dimensions of the expander. Small- and micro-power turbines feature very high rotational speeds, which leads to complications in the design of the clutch connecting the expander and electrical generator.

Compared to micro turbines, volumetric expanders feature a lower range of operating pressure and lower gas flow capacity. In the prototypes of small and micro-power ORC systems, scroll, piston and other types of volumetric expanders are adopted. Scroll expanders are complex in design, their manufacturing requires advanced engineering facilities in order to maintain the high quality of scrolls workmanship, which directly translates into the high price of such expanders. Prototypes of scroll expanders adopted in ORC systems are manufactured in oil-free versions. Compared to the scroll expander, piston expander has a much simpler design, but requires lubrication, valve timing, and as a result of its cyclical operation, vibrations are generated.

In turn, the multi-vane expander design is very simple, which translates into low production costs. This machine features an advantageous ratio of the power output to the external dimensions. The application of special construction materials gives the opportunity for eliminating the need for lubrication. Compared to the other types of volumetric machines and micro turbines, multi-vane machines features a lower gas flow capacity and lower expansion ratios. Moreover, it can be easily hermetically sealed, which is one of the key issues in the ORC system design. Multi-vane machines are insensitive to the 
negative influence of the gas-liquid mixture expansion which, in the case of powering ORC systems by heat sources featuring floating thermal characteristics, is a great advantage. Multi-vane compressors and expanders are applied in many industrial applications, including mining, refrigeration and pneumatic systems. Multi-vane expanders feature power outputs of several hundred $\mathrm{W}$ to approximately $5 \mathrm{~kW}$. Maximum gas pressure on the inlet to multi-vane expander reaches approximately 10 bar. These machines feature rotational speeds of $3000 \mathrm{rev} / \mathrm{min}$.

The above described features of multi-vane expanders indicate that these machines may be a promising alternative to micro turbines and other volumetric expanders applied in small- and micro-power CHP ORC prototypes designed for domestic generation of electricity and useful heat by prosumers. The literature review shows issues that are insufficiently recognized and scientifically described related to: operating of multi-vane expander with different low-boiling working fluids, internal leakages of working fluid in expanders' working chambers, design of the inlet and outlet ports, optimization of the expander geometry, lowering the expanders' internal friction and a gas-liquid mixture expansion. The solution of the raised problems requires comprehensive study and experimental analysis. Thus, the authors decided to carry out the experimental and numerical study on these issues. In the following part of this paper the description of the experimental test-stand, the multi-vane expander and the experimental results together with the description and results of numerical analysis are presented. The numerical investigations showed the insight in the flow phenomena in the expanders' working chambers and indicated the differences in these phenomena for analysed working fluids. Moreover, obtained results showed the expanders' design imperfections, i.e. sharp edges of the inlet and outlet ports causing working medium turbulence and internal leakages, reducing expanders' output power. The numerical model was validated with experimental data what proved its suitability and allowed to use the model in testing modifications to the machines design or finding the optimum working parameters and working fluid. In the paper a numerically obtained results of the multi-vane expander operation for various working media is presented. All refrigerants were modelled for a range of pressure and temperature parameters to find the optimal working point for the Organic Rankine Cycle.

\section{Description of the experimental test-stand, the multi-vane expander and the experiment results}

The experimental investigations were carried out using a prototype of domestic combined heat and power (CHP) ORC system featuring the thermal power of $18 \mathrm{~kW}$ and electric power of $300 \mathrm{~W}$. A general view of the test-stand is presented in Fig. 1.

The main test-stand components are: a plate evaporator (1), a plate condenser (2), a working fluid pump (3), a reservoir of a working fluid (4), and a micro-vane expander (5). The working fluid is R123. The heat source for the test-stand is hot water (heated in a gas boiler) which temperature can be regulated in the range of $40-95{ }^{\circ} \mathrm{C}$. The condenser is cooled by the cold water in the open cycle.

The working principle of the test-stand can be described as follows. At first, the hot water is pumped from the gas boiler to the evaporator and circulates between the plates. Then, the liquid R123 is pumped from the reservoir to the evaporator where it is evaporated. Obtained pressurized vapor drives the multi-vane expander. After the expansion, the vapor is cooled and liquefied in the condenser by the cold water circulating between the plates. At last, liquefied working fluid flows to the reservoir and the cycle is therefore complete.

The applied expander is an air motor which was adapted for low-boiling working fluid by application of stainless steel bearings, PTFE seals and graphite vanes. The expander 
features the maximal power of $300 \mathrm{~W}$. The main expander components are: the cylinder, the rotor, vanes and two end covers. The gas is supplied to the expanders' working chambers through the inlet port and flows out of the expander through the outlet port. Fig. 2 shows a general view of the expander. Detailed description of the expander is presented in [3].

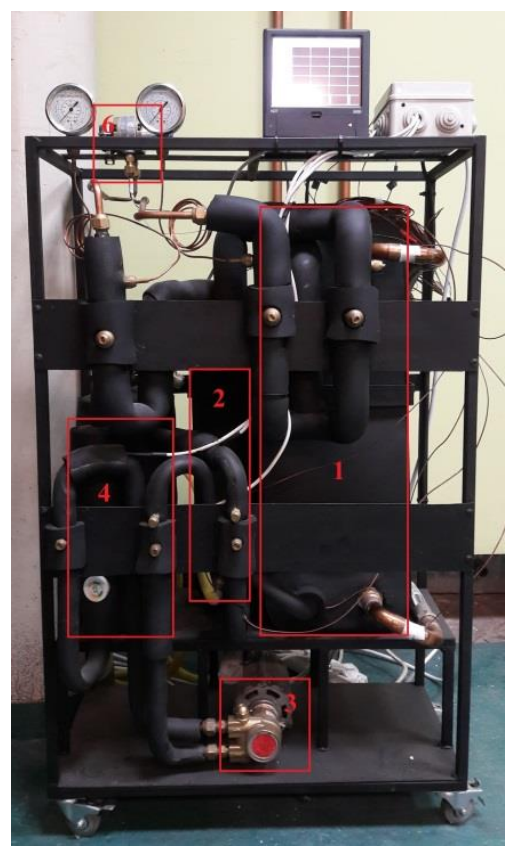

Fig. 1. A general view of the test-stand.

1 - plate evaporator, 2 - plate condenser, 3 - working fluid pump, 4 - working fluid reservoir, 5 multi-vane expander.

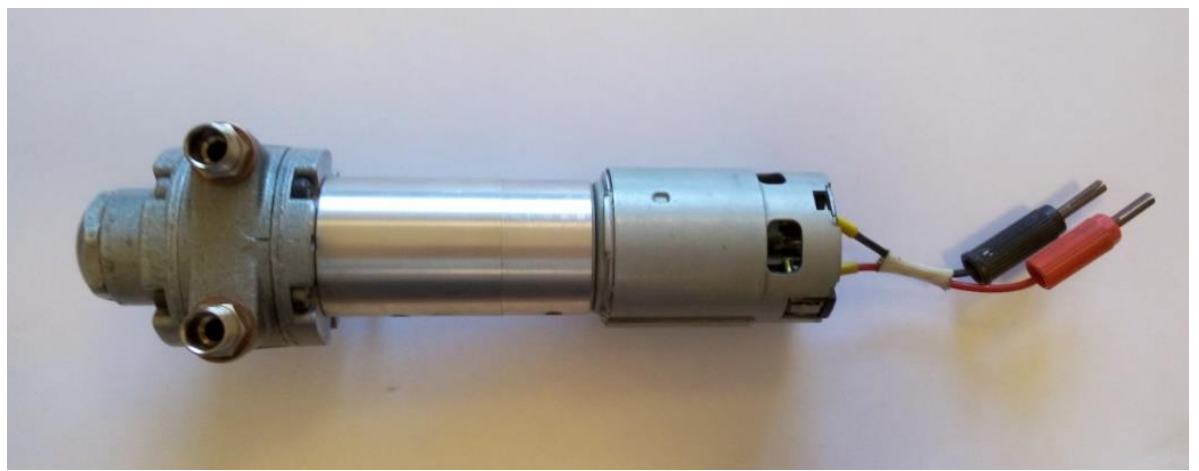

Fig. 2. A general view of the expander.

In order to obtain the experimental data needed for validation of expanders' numerical model, a series of experiments with varied measurement conditions were carried out using the test-stand.

The experimental series were performed for the values of the heat source temperature ranging between $55^{\circ} \mathrm{C}$ and $85^{\circ} \mathrm{C}$ and for the working fluid pressure ranging between 2.0 and 5.2 bar. The experimental results showed that the expander indicated work varies in the range of $0.96-4.18 \mathrm{~kJ} / \mathrm{kg}$ while its internal efficiency varies in the range of $17.2-58.3 \%$ 
depending on the experimental conditions. The more detailed description of the experiment and experimental results is presented in [3]. The results from the experiment were in a good agreement with the numerical predictions so the model was validated for a given range of temperature and pressure parameters.

\section{Numerical modelling}

\subsection{Description of the numerical model}

A transient operation of the multi-vane expander was modeled numerically using transient boundary conditions. The model complies the rotational motion of the rotor and the vanes as well as the vanes radial position change during the process. This allows for an in-depth calculation of the working medium inside a working chamber including the internal leakages. The inlet and the outlet pipes remain stationary and they relate to the rotors domain through the interface (see Figure 3).

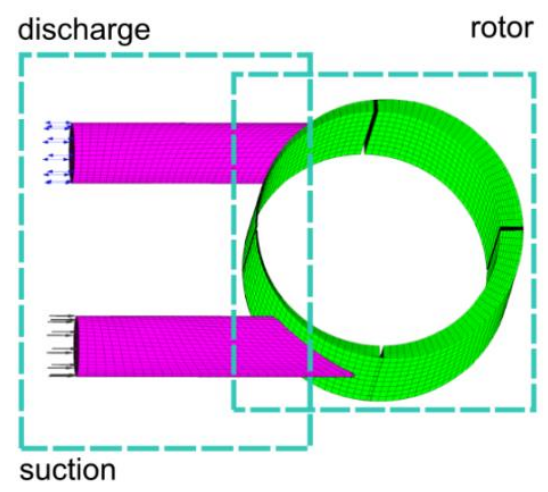

Fig. 3. A numerical mesh of the numerical model.

The working fluid flow was modelled as a compressible, turbulent and single-phase ideal gas. The specific heat capacity of each tested medium was set as a function of temperature. The wall boundary was assumed adiabatic due to the short time spent by a portion of the fluid inside the machine (see Table 1).

Tab. 1. Boundary conditions and model assumptions

\begin{tabular}{|c|c|c|}
\hline Parameter & Value & Remarks \\
\hline Inlet temperature & $40-90^{\circ} \mathrm{C}$ & with step of 10 degrees \\
\hline Inlet pressure & $\begin{array}{c}\text { Saturation pressure at the inlet } \\
\text { temperature }\end{array}$ & - \\
\hline Condenser Temperature & $20^{\circ} \mathrm{C}$ & $\begin{array}{c}\text { depending on the } \\
\text { refrigerant }\end{array}$ \\
\hline Condenser Pressure & Saturation pressure at $20^{\circ} \mathrm{C}$ & 1 degree of revolution \\
\hline Time step & $5.5 \times 10^{-5} \mathrm{~s}$ & 5 full revolutions \\
\hline Total time & $0.1 \mathrm{~s}$ & Standard model \\
\hline Turbulence model & $k$-epsilon & - \\
\hline Wall heat transfer & Adiabatic & constant \\
\hline Vane-to-wall clearance & $40 \mu \mathrm{m}$ &
\end{tabular}


Parameters at the inlet to the expander were selected to be temperature and pressure. For each working fluids the inlet temperature was varied in a range of $40-90^{\circ} \mathrm{C}$ with step of 10 degrees. The inlet pressure was taken as a saturation pressure of the working fluid at the inlet temperature.

The rotor rotational speed was assumed constant at a nominal value of $3000 \mathrm{rpm}$ for all considered cases. The temperature at the outlet of the discharge socket was assumed $20^{\circ} \mathrm{C}$ for all the cases and the pressure was set to the saturation pressure given by the medium properties.

\section{Results and discussion}
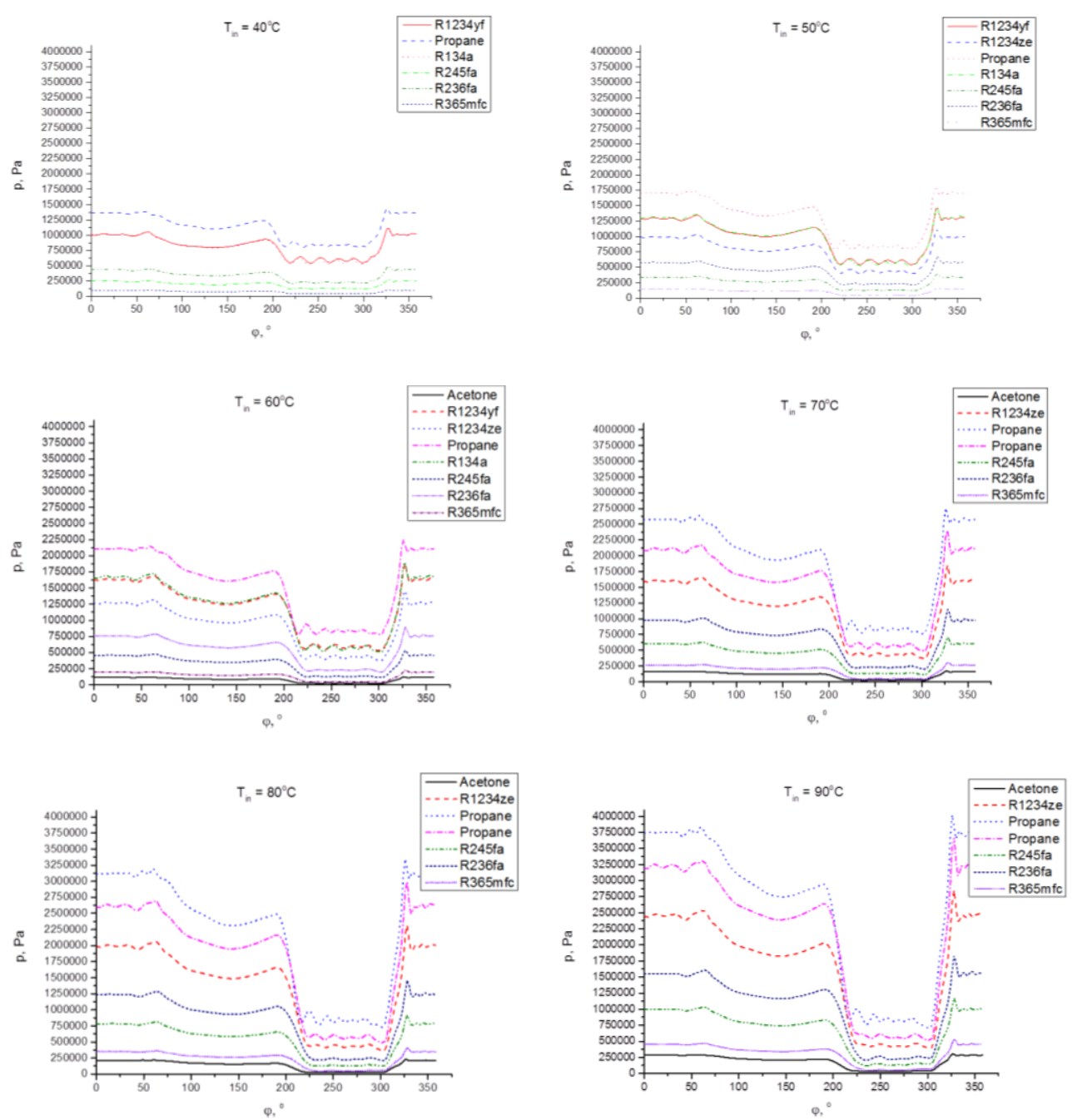

Fig. 4. Pressure distribution against rotational angle for different working fluids and inlet temperatures.

In Fig. 4 pressure distribution in a working chamber versus rotational angle, for different working fluids and different inlet temperatures, is presented. With increasing the 
inlet temperature the inlet pressure (i.e. saturation pressure) increases and the area under the curve in the $p-\varphi$ diagram increases. Moreover the saturation pressure differs for each working fluid. The highest inlet pressure occurred for propane and the lowest for acetone. Presented curves show a standard variation except the strange recompression effect which is visible in the vicinity of $\varphi=200^{\circ}$. This behaviour stems from the improper construction of the expander and it was described in detail in [3]. This effect is more visible for working fluids with a higher saturation pressure and it results in decrease of efficiency of the expander.

In order to better understand the influence of a working fluid on the expander's performance, indicated work was calculated as a difference between the inlet and the outlet enthalpy. Fig. 5 shows the results of indicated work versus pressure ratio for working fluids used. Almost all curves (except the one for acetone) characterize of a similar shape, i.e. they first steeply increase achieving the maximum in the range of $\sigma=3-4$ (only propane for $\sigma \approx 2$ ) and then gradually decrease with increasing $\sigma$. Different behaviour presents the curve for acetone where the minimum is visible for $\sigma \approx 6,5$. From these results one can infer that optimal pressure ratio for the rotary vane expander is located in the range of $\sigma=$ 3-4 and the best performance can be accomplished with propane for $\sigma \approx 2$. However, more detailed investigation of the results is shown in Fig. 6 where the efficiency of the expander versus pressure ratio is plotted. It is obvious now that using propane as a working fluid in the expander analysed is very ineffective and features very low efficiency of the order of $5 \%$. However, Fig. 6 also confirms that the optimal pressure ratio is located in the range of $\sigma=3-4$ for which the efficiency is in the range of $12-35 \%$. The highest efficiency can be achieved for the acetone, however the pressure ratio has to be increased to about $\sigma \approx 5$.

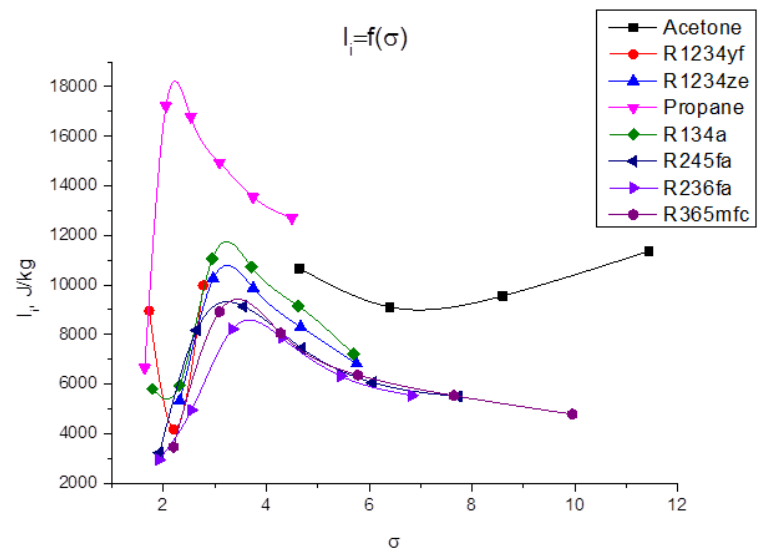

Fig. 5. Indicated work against pressure ratio for different working fluids. 


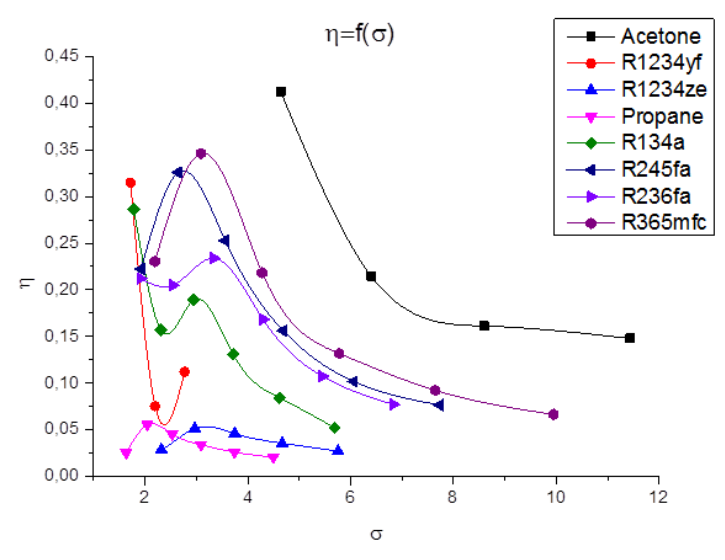

Fig. 6. Efficiency against pressure ratio for different working fluids.

\section{Summary and conclusions}

In the paper the numerical investigation of rotary vane expander was presented. The experimental survey was carried out using a prototype of domestic ORC system equipped with small multi-vane expander featuring a power of $300 \mathrm{~W}$ and utilizing R123 as a working fluid. The main aim of experimental investigations was gathering the input data needed for creating the numerical model of the expander and analysis of its operating conditions with a variety of working fluids. Additionally, during the experiment the operation of the expander was kept under observation. It was found that the expander was operating continuously and without serious problems. The experimental results confirmed that multi-vane expanders are suitable to small power domestic ORC systems and proved the usability of the numerical model.

The numerical survey on the expander operation was carried out for different working fluids i.e. R1234yf, R1234ze, R134a, R245fa, R236fa, R365mfc, Acetone and Propane. During the numerical simulations the value of temperature on the expanders' inlet was varied similarly as during the experiments. The results of numerical analysis showed that for the expander analysed and working fluids used, the optimal pressure ratio is in the range of $\sigma=3-4$. Corresponding efficiency is in the range of $12-35 \%$ depending on working fluid. This results confirms the findings of survey presented in [4], i.e. the multi-vane expanders feature the highest power and efficiency within this certain range of pressure ratios. Thus, increasing the pressure ratio is not profitable and results in decreasing the power and efficiency of the expander. Additionally it can be concluded that the domestic ORC systems adopting multi-vane expanders should be filled with working fluids featuring moderate pressures at the considered level of the heat source temperatures $\left(40-90^{\circ} \mathrm{C}\right)$.

\section{References}

1. T.C. Hung, T.Y. Shai, S.K. Wang, Energy 22, 661-667 (1997)

2. P. Kolasiński, Energies 8, 3351-3369 (2015)

3. P. Kolasiński, P. Błasiak, J. Rak, Energies 9, 606 (2016)

4. Z. Gnutek, P. Kolasiński, J Eng Gas Turb Power Journal 135, 061901 (2013) 